\title{
Can Peroperative Neutrophil To Lymphocyte Ratio Change (Deltanlr) Be Used as a Parameter in Predicting Acute Renal Failure Following Coronary Bypass Operations With Cardiopulmonary Bypass?
}

\author{
Sefer Usta, MD, ${ }^{1}$ Mustafa Abanoz, MD $^{2}$ \\ ${ }^{1}$ University of Health Sciences, Ahi Evren Thoracic and Vascular Surgery Training and Research Hospital, Department of \\ Cardiovascular Surgery, Trabzon, Turkey; ${ }^{2}$ University of Health Sciences, Mehmet Akif İnan Training and Research Hospital, \\ Department of Cardiovascular Surgery, Karaköprü, Şanlıurfa, Türkiye
}

\section{ABSTRACT}

Background: Inflammation plays a significant role in the pathogenesis of many diseases as well as postoperative acute renal failure (ARF). Preoperative neutrophil to lymphocyte ratio (NLR) values have a prognostic value for postoperative ARF after cardiovascular surgeries.

Methods: Patients who underwent elective coronary artery bypass graft (CABG) with cardiopulmonary bypass in our clinic between December 15, 2015 and December 15, 2019, retrospectively were included in this study. Patients who did not develop ARF after the operation were categorized as Group 1, and patients who did were included in Group 2. NLR was calculated from the hemograms during three periods (Preoperative (Pre), Postcardiotomy (Pc), Postoperative Day 1 (Po1). DeltaNLR1 (PcNLR- PreNLR) and DeltaNLR2 (Po1NLR-PreNLR) values were obtained from these calculated values.

Results: The mean ages of patients in Group $1(\mathrm{~N}=274)$ and Group $2(\mathrm{~N}=61)$ were $60 \pm 9.1$ years and $67.7 \pm 9.8$ years, respectively $(P<.001)$. In the multivariate analysis, being over 65 years of age (Odds ratio [OR]: 1.074, 95\% confidence interval [CI]: 1.012-1.194, $P=.030)$, postoperative inotropic need (OR: 0.678, CI 95\%: 0.395-0.819, $P=.021$ ), increased blood product use (OR: 0.916, CI 95\%: 0.779-0.986, $P=.034)$, preoperative creatinine increase (OR: 1.974 , CI 95\%: 1.389-4.224, $P=.007$ ), PcNLR (OR : 1.988, CI 95\%: $1.765-3.774, P<.001)$, Po1NLR (OR: 1.090 , CI 95\%: $1.007-$ 2.116, $P=.028$ ), DeltaNLR1 (OR: 3.090 , CI 95\%: $1.698-$ $6.430, P<.001$ ) and DeltaNLR2 (OR: 1.676 , CI 95\%: $1.322-$ $2.764, P=.003)$ were identified as independent predictors for predicting postoperative ARF.

Conclusion: In this study, we have shown that peroperative NLR changes can be used as an effective parameter to predict ARF developing following CABG operations.

Received October 23, 2020; accepted November 18, 2020.

Correspondence: Mustafa Abanoz, MD, University of Health Sciences, Mehmet Akif Inan Training and Research Hospital, Department of Cardiovascular Surgery, Esentepe Mah. Ertuğrul Cad, 63200, Karaköprü/Şanhurfa, Türkiye; +90414-318-00-00; fax: +90414-318-67-07 (e-mail: mustafaabanozdr@ hotmail.com).

\section{INTRODUCTION}

Coronary artery bypass graft (CABG) surgery is a commonly used treatment method for coronary artery disease (CAD). While facilitating the procedure, the use of the cardiopulmonary bypass (CPB) system in this surgery also carries several risks [Engin 2020]. With this system, inflammatory pathways are activated due to the contact of blood with outer surfaces, thus morbid conditions can develop. One of the most important of these morbid conditions is renal failure. The rate of acute renal failure (ARF) is around 5-30\% following open heart surgery, and renal replacement therapy may be required in 1-2\% of these patients [Takaki 2015]. Many factors, such as cardiopulmonary bypass times, advanced age, preoperative renal failure and blood transfusion, play a role in the occurrence of this condition [Freeland 2015; Parlar 2018; Tumer 2019].

Inflammation plays a significant role in the pathogenesis of many diseases as well as postoperative ARF. Neutrophil lymphocyte ratio (NLR) is an important parameter that can be obtained easily and cheaply from inflammatory parameters. Its prognostic significance for renal failure has been demonstrated in patients with severe sepsis [Yilmaz 2015]. In recent studies, it has been revealed that preoperative NLR values have a prognostic value for postoperative ARF after cardiovascular surgeries [Parlar 2018; Kim 2015]. However, in some studies from various fields of medicine, including the cardiovascular field, it has been found that changes in NLR values (DeltaNLR) before and after the procedure carry a prognostic value [Li 2017; Seong 2019].

In this study, we aimed to investigate the importance of preoperative NLR changes in predicting ARF after CABG operations accompanied by CPB.

\section{MATERIALS AND METHODS}

Patients who underwent elective CABG with CPB in our clinic between December 15, 2015 and December 15, 2019, were included in this study, which started after the approval of the local ethics committee. The study was conducted, according to the principles of the Declaration of Helsinki. Written informed consent forms were obtained from all 
Table 1. Demographic datas and peroperative features of the patients

\begin{tabular}{|c|c|c|c|}
\hline Age (years) & $60 \pm 9.1$ & $67.7 \pm 9.8$ & $<.001 \dagger$ \\
\hline Hypertension, n (\%) & $155(56.5 \%)$ & $45(73.7 \%)$ & $.012 *$ \\
\hline COPD, n (\%) & $54(19.7 \%)$ & $13(21.3 \%)$ & $.684 *$ \\
\hline Previous PCl, n (\%) & $70(25.5 \%)$ & $21(34.4 \%)$ & $.239 *$ \\
\hline Angiography to operation time, days & $3(1-70)$ & $3(1-90)$ & $.257 \ddagger$ \\
\hline Current smoker, $\mathrm{n}(\%)$ & $58(21.1 \%)$ & $15(24.5 \%)$ & $.696^{*}$ \\
\hline $\mathrm{BMI}\left(\mathrm{kg} / \mathrm{m}^{2}\right)$ & $27.4(23-39.5)$ & $28.6(22.9-40)$ & $.357 \ddagger$ \\
\hline Hiperlipidemia, n (\%) & $81(29.5 \%)$ & $21(34.4 \%)$ & $.554 *$ \\
\hline
\end{tabular}

*Chi-square test, †Student’s $t$ test (Data is axpressed as mean $\pm s d$ ), $\ddagger$ Mann Whitney $U$ test (Data is expressed as median (interquartile range). BMI, body mass index; COPD, chronic obstructive pulmonary disease; PCl, percutaneus coronary intervention; ASA, acetylsalicylic acid; ACEl, angiotensin-converting enzyme inhibitor; ARB, angiotensin receptor blocker

patients before their surgeries. Emergency operations, combined surgeries, those with renal failure in the preoperative period (creatinine $>1.5 \mathrm{mg} / \mathrm{dl}$ ), those who have had a percutaneous coronary intervention in the past month, those with systemic inflammatory disease, thyroid dysfunction, those who were re-operated due to bleeding or preoperative myocardial infarction, and patients who received steroid treatments were excluded from the study. Consequently, 335 patients were included. The data of the patients were accessed from the hospital registration system and intensive care unit daily observation cards. Demographic and preoperative features (age, sex, smoking, hypertension, diabetes mellitus, routine blood parameters, etc.), operative (cardiopulmonary bypass times, graft counts, total blood product use), and postoperative data (routine blood parameters, total blood product use, hospital and intensive care hospitalization times, etc.) were recorded. Patients who did not develop ARF after the operation were categorized as Group 1, and patients who did were included in Group 2.

Definition of postoperative renal failure: All patients included in the study had preoperative creatinine values below $1.5 \mathrm{mg} / \mathrm{dl}$. The development of intra-hospital ARF in patients was defined as the primary endpoint of the study. Renal failure after the operation was determined according to Kidney Disease Improving Global Outcomes (KDIGO) criteria, which is staged as shown below [Parlar 2018].

Stage 1: Serum creatinine increase of $\geq 0.3 \mathrm{mg} / \mathrm{dl}$ or 1.5 to 1.9 times the value of baseline.

Stage 2: Serum creatinine increased to 2.0 to 2.9 times the value of baseline.
Stage 3: Serum creatinine increased to 3.0 or more times of baseline or $\geq 4.0 \mathrm{mg} / \mathrm{dl}$ or commencement of renal replacement therapy.

Patients who developed any of the above-mentioned intrahospital stages were considered as having ARF.

Calculation of neutrophil lymphocyte ratio and changes: In our hospital, all patients undergoing cardiac surgery are evaluated with hemograms preoperatively, postoperatively at arrival in the intensive care unit, and for three consecutive days after that. NLR was calculated from the hemograms during three periods (Preoperative (Pre), Postcardiotomy (Pc), Postoperative Day 1 (Po1). DeltaNLR1 (PcNLR- PreNLR) and DeltaNLR2 (Po1NLR-PreNLR) values were obtained from these calculated values.

Statistical analysis: Statistical analysis of the data was performed with SPSS 21.0 (IBM Statistical Package for the Social Sciences Statistic Inc. version 21.0, Chicago, IL, USA). Ordinal and continuous data were expressed in mean and standard deviation. Normality of distribution was assessed with Kolmogorov-Smirnov and Shapiro-Wilk tests. To evaluate the normally and non-normally distributed data, Student's t-test and Mann Whitney U tests were used, respectively. Nominal variables were analyzed for frequency and percentage, after which they were compared using the Chi-Square test. ARF predictors were evaluated with multivariate binary logistic regression analysis. The predictive values of preoperative NLR, DeltaNLR1, and DeltaNLR2 in ARF were determined with Receiver Operating Characteristic (ROC) curve analysis and calculation of Area Under Curve (AUC). A $P$ value <.05 was considered significant. 
Table 2. Preoperative laboratory variables of the patients

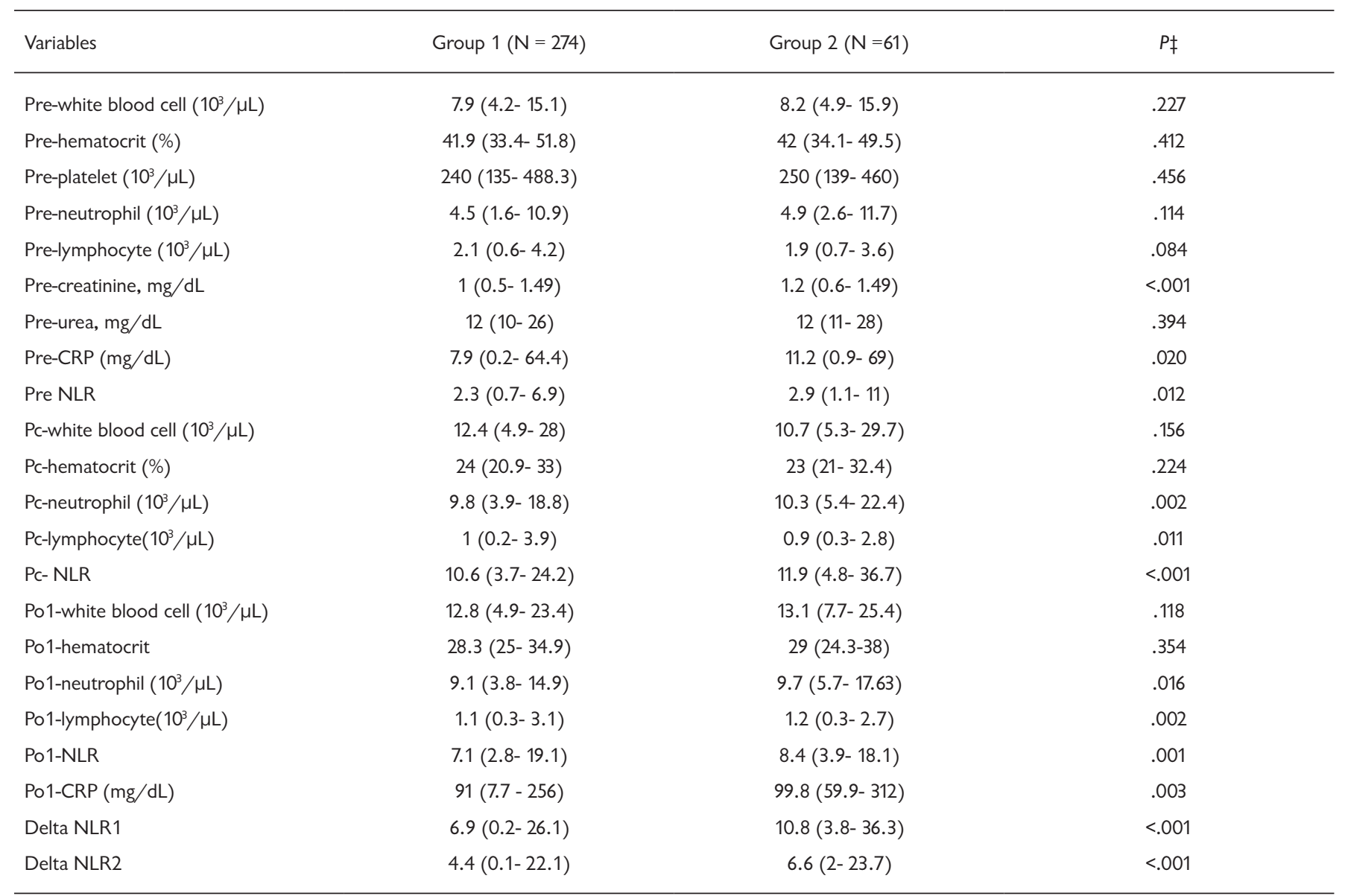

$\ddagger$ Mann-Whitney $U$ test (data expressed as median with interquartile range), CRP, C-reactive protein; NLR, neutrophil to lymphocyte ratio; Pre, preoperative; Pc, postcardiotomy; Po1, postoperative day 1

\section{RESULTS}

A total of 335 patients were included in the study. Postoperative ARF developed in 61 (18.2\%) patients. According to the KDIGO classification of patients with ARF (Group 2), 29 $(47.5 \%)$ were in Stage-1, 25 (40.9\%) were in Stage-2, and 7 $(11.4 \%)$ were in Stage-3. The mean ages of patients in Group $1(\mathrm{~N}=274)$ and Group $2(\mathrm{~N}=61)$ were $60 \pm 9.1$ years and 67.7 \pm 9.8 years, respectively $(P<.001)$. The groups were similar in terms of gender, body mass index, hyperlipidemia, hypertension (HT), chronic obstructive pulmonary disease (COPD), and current medical treatments (Acetylsalicylic acid, angiotensin receptor blocker therapy). The rates of diabetes mellitus (DM) and hypertension (HT) were significantly higher in Group $2(P=.019$ and $P=.012$, respectively) (Table 1).

The peroperative laboratory parameters of the patients are presented in Table 2. There were no significant differences between the groups, in terms of preoperative hemogram parameters and biochemical parameters such as total protein, albumin, and urea. Preoperative $\mathrm{C}$ reactive protein (CRP), creatinine and NLR values were significantly higher in Group 2
( $P=.020, P<.001$, and $P=.042$, respectively). In the postcardiotomy period, neutrophil and NLR values were significantly higher in Group 2 and lymphocyte values were significantly lower $(P=.002, P=.011$, and $P<.001$, respectively). On the first postoperative day, neutrophil, CRP, and NLR values were significantly higher in Group 2 and lymphocyte values were significantly lower $(P=.016, P=.003, P=.001$, and $P=.002$, respectively). Calculated DeltaNLR1 and DeltaNLR2 values were also significantly higher in Group $2(P<.001)$.

Other peroperative variables of the patients are presented in Table 3. Total perfusion time, total amount of blood product use, inotropic requirement rate, intensive care, and total hospital stay were significantly higher in Group $2(P=.014$, $P=.002, P=.001, P<.001$, and $P<.001$, respectively).

In ROC analysis, cut-off values were $9.1(80 \%$ sensitivity, 66.7\% specificity) for Delta NLR1, 5.6 (71\% sensitivity, 63.5\% specificity) for Delta NLR2, 9.7 (78.5\% sensitivity, 65.5\% specificity) for PcNLR and 7.9 (74.2\% sensitivity, $62.4 \%$ specificity) for Po1NLR (Figures 1 and 2).

Logistic regression analysis was performed to predict ARF after the operation (Table 4). In the multivariate analysis, 
Table 3. Peroperative variables of the patients

\begin{tabular}{lccl}
\hline Variables & Group 1 $(\mathrm{N}=274)$ & Group 2 (N=61) & $.014 \ddagger$ \\
\hline Total perfusion time & $98(52-178)$ & $108(55-195)$ & $.364 \ddagger$ \\
Cross-clamp time & $80(33-100)$ & $84(36-116)$ & $.614 \ddagger$ \\
Number of distal anastomoses & $3(1-5)$ & $3(1-6)$ & $.002 \ddagger$ \\
Packed blood products (units) & $5(5-11)$ & $6(4-15)$ & $.001^{*}$ \\
Inotropic support, $\mathrm{n}(\%)$ & $59(21.5 \%)$ & $25(40.9 \%)$ & $<.001 \ddagger$ \\
Total ICU stay (days) & $2(2-5)$ & $4(2-19)$ & $<.001 \ddagger$ \\
Total hospital stay (days) & $7(6-12)$ & $11(6-33)$ & \\
\hline
\end{tabular}

* Chi-square test, $\ddagger$ Mann Whitney $U$ test (Data is expressed as median (interquartile range), ICU, intensive care unit

being over 65 years of age (Odds ratio [OR]: 1.074, 95\% confidence interval $[\mathrm{CI}]: 1.012-1.194, P=.030)$, postoperative inotropic need (OR: 0.678, CI 95\%: 0.395-0.819, $P=.021$ ), increased blood product use (OR: 0.916, CI 95\%: 0.779-0.986, $P=.034)$, preoperative creatinine increase (OR: 1.974 , CI 95\%: 1.389-4.224, $P=.007$ ), PcNLR (OR : 1.988, CI 95\%: 1.765-3.774, $P<.001$ ), Po1NLR (OR: 1.090 , CI 95\%: $1.007-$ 2.116, $P=.028$ ), Delta NLR1 (OR: 3.090, CI 95\%: 1.698$6.430, P<.001$ ) and DeltaNLR2 (OR: 1.676 , CI 95\%: $1.322-$ $2.764, P=.003)$ were identified as independent predictors for predicting postoperative ARF.

\section{DISCuSSION}

Our research revealed that the values of NLR, which is considered a valuable parameter in the progression and prognosis of cardiovascular diseases, obtained during the postcardiotomy period and on the first postoperative day following CABG with $\mathrm{CPB}$, are independent predictors of in-hospital ARF. Also, to the best of our knowledge, we have shown for the first time in the literature that NLR difference before and after CABG operations is predictive for ARF. In the multivariate analysis performed in our study, we determined the DeltaNLR1 value was predictive compared to other parameters (OR: 3.090, CI 95\%: 1.698-6.430, $P<.001$ ).

Coronary artery bypass surgery is currently performed with very low mortality and morbidity rates. ARF, which occurs after these operations, is an undesirable condition that may affect the surgical results and prolong the treatment process. Ischemia reperfusion injury and inflammation are critical factors in the development of ARF. As a result of an acute ischemic event in the renal endothelium, adhesion molecules are activated and inflammatory blood cells adhere to the endothelium, leading to possible renal damage [Bonventre 2011; Sharfuddin 2011]. NLR is one of the most commonly used inflammatory parameters because it is easily available and inexpensive. After coronary bypass operations, the inflammatory response mainly develops with cellular immune system activation, which is based on lymphocytes. As a result of this activation, neutrophil counts increase and lymphocyte counts decrease [Laffey 2002; Erdolu 2020], resulting in elevated NLR values, an important parameter showing inflammation.

In a retrospective observational study conducted by Kim et al, the importance of NLR value in predicting ARF was investigated in 590 patients undergoing open heart surgery, among which postoperative ARF rate was reportedly $28.1 \%$. The authors calculated the NLR values during the same three periods with ours. Akin to our study, since the preoperative NLR value was used as a continuous parameter, it was not found significant in predicting ARF. PcNLR value was found to significantly correlate with ARF when used as a continuous parameter. In the multivariate analysis, $\mathrm{PcNLR}$ value was evaluated by dividing it into four quartiles and PcNLR $\geq 10$ was found the most predictive value among all analyzed NLR values (OR: 5.90, 95\% CI: 2.74-12.73, $P$ < .001) [Kim 2015]. Although our study included differences in terms of involving isolated CABG patients, among all NLR values we calculated, we found the PcNLR value more predictive of ARF (OR: 1.988, CI 95\%: 1.765-3.774, $P<.001)$. In addition, we determined the cut-off value for PcNLR as 9.7 (78.5\% sensitivity, $65.5 \%$ specificity) in the ROC curve analysis.

The importance of the NLR value was investigated in predicting ARF occurring after CABG operations by Parlar et al. A total of 311 patients were included in the study, which was designed retrospectively, and the rate of ARF was found to be $19.9 \%$ in the postoperative period. Unlike our study, NLR values were calculated in preoperative, postoperative days 1,3 and 7. In multivariate analysis, the most predictive NLR value was determined as that obtained on the first postoperative day (OR: 3.04, CI 95\%: 1.51-6.10, P=.002) [Parlar 2018], as in our study, in which the NLR value measured on the first day after the operation was an independent predictor for ARF (OR: 1.090, CI 95\%: 1.007-2.116, $P=.028$ ). However, we did not detect the preoperative NLR value as a predictor for ARF. Weedle et al also did not determine preoperative NLR values to be effective on ARF and found that NLR values calculated on the first and second postoperative days correlated with ARF (OR: 1.02, CI 95\%: 1.004-1.03, $P=.015$, OR: 1.06, CI 95\%: 1.03-1.10, $P<.001$, respectively) [Weedle 2019].

Studies performed in recent years state that it is the change of these parameters before and after the surgery, rather than 
Table 4. Logistic regression analysis to identify factors affecting postoperative acute renal failure

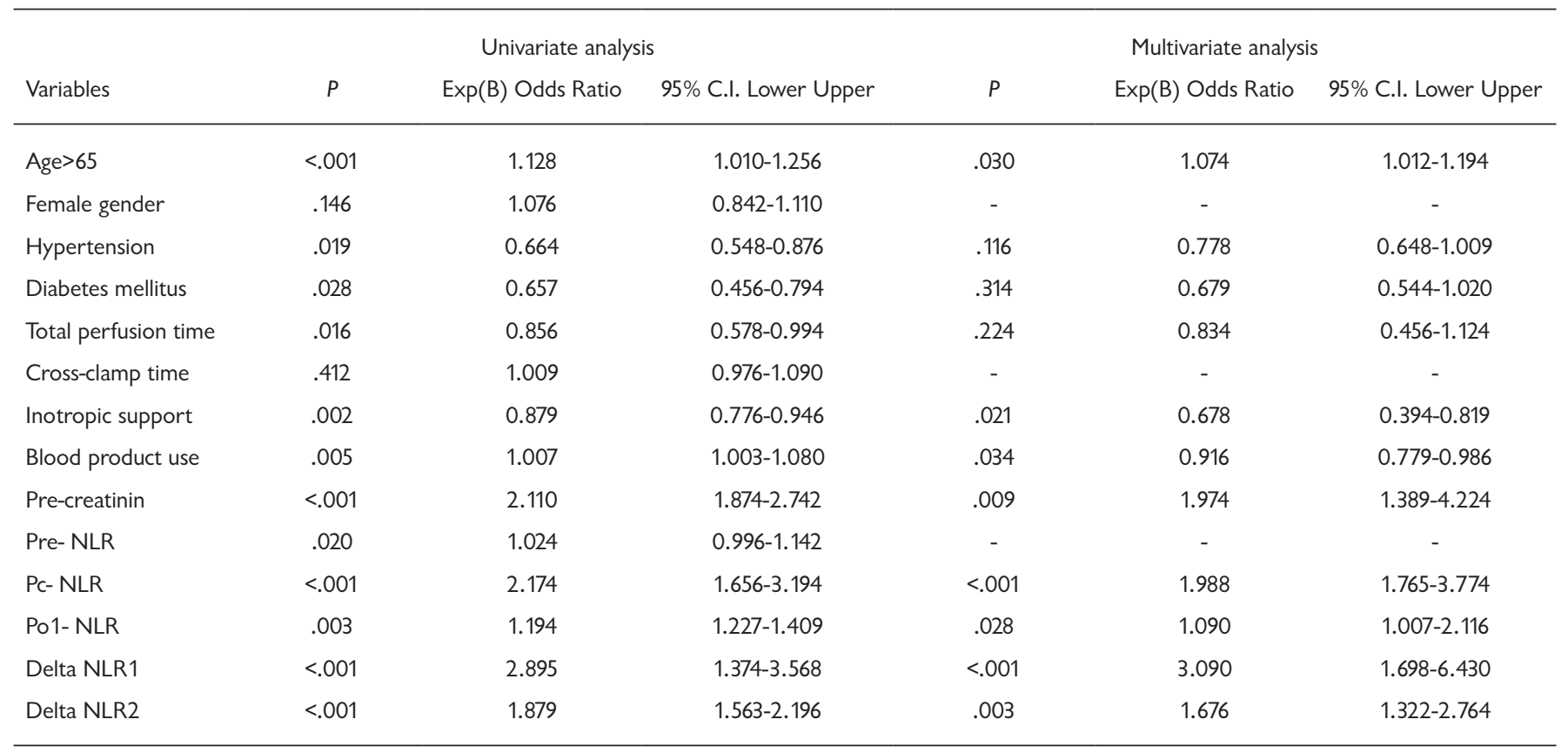

COPD, chronic obstructive pulmonary disease; CRP, C-reactive protein; NLR, neutrophil to lymphocyte ratio; Pre, preoperative; Pc: postcardiotomy; Po1: postoperative day 1

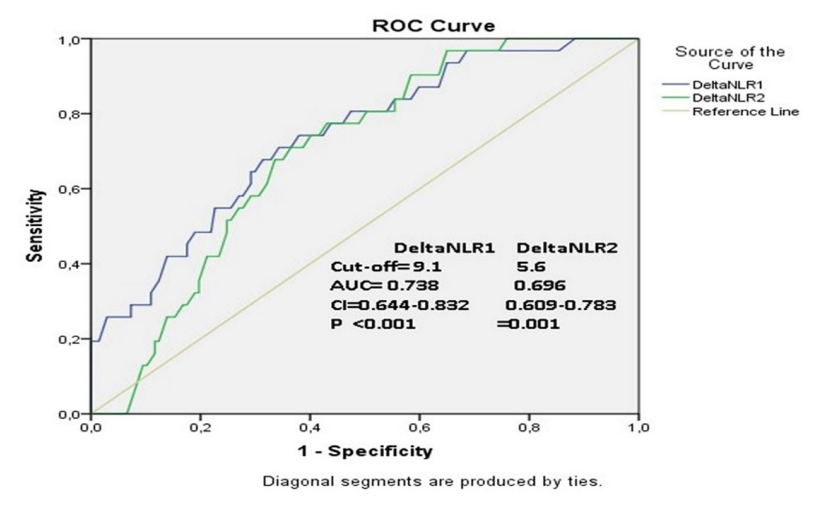

Figure 1. Data figure of the area under the curve (AUC), confidence interval $(\mathrm{Cl})$ and cut-off values in receiver operating characteristic curve analysis for DeltaNLR1 (80\% sensitivity, $66.7 \%$ specificity) and DeltaNLR2 (71\% sensitivity, $63.5 \%$ specificity).

the parameters obtained from routine blood tests, which may be of prognostic value. For example, the effects of changes in red blood cell distribution (RDW) on mortality after CABG operations were investigated by Lee et al, and DeltaRDW value was determined as an independent predictor in predicting early postoperative adverse events in multivariate analysis [Lee 2018]. DeltaNLR value has recently been investigated in various fields of medicine in this respect. Seong et al investigated the importance of DeltaNLR in predicting prognosis after primary pulmonary sarcomatoid carcinoma surgery

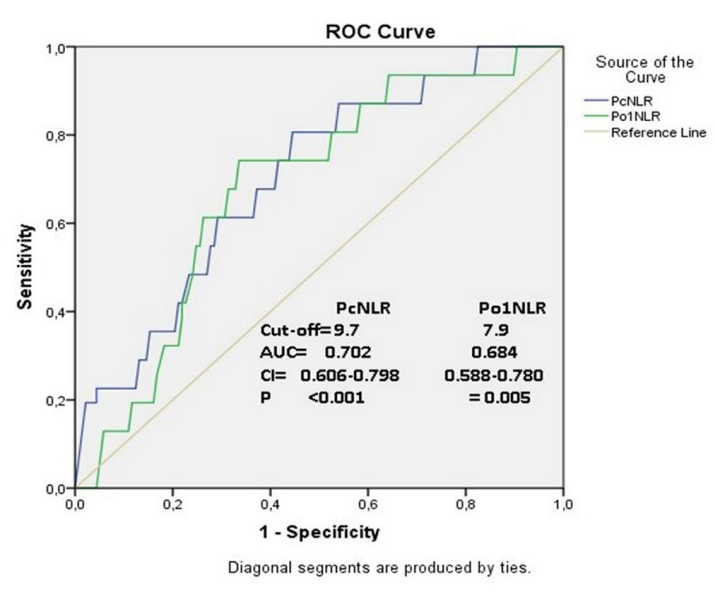

Figure 2. Data figure of the area under the curve (AUC), confidence interval $(\mathrm{Cl})$ and cut-off values in receiver operating characteristic curve analysis for PcNLR (78.5\% sensitivity, 65.5\% specificity) and Po1NLR (74.2\% sensitivity, $62.4 \%$ specificity).

and calculated DeltaNLR value by subtracting the NLR value before the operation from the NLR value immediately after the operation. Authors found that increased DeltaNLR values were associated with poor postoperative results [Seong 2019]. The study investigating the place of DeltaNLR in cardiovascular diseases was conducted by $\mathrm{Li}$ et al, in which the importance of DeltaNLR was evaluated in predicting the major adverse cardiovascular events occurring after 
percutaneous coronary intervention in patients with chronic total occlusion. DeltaNLR value was calculated as the difference between the value before and after the procedure. At the end of the study, the authors determined the DeltaNLR value as an independent predictor in predicting restenosis development (OR: 2.538, CI 95\%: 1.019-5.508, $P=.049)$. In addition, 1-year major adverse cardiovascular events were found to be significantly higher in patients with high DeltaNLR values [Li 2017]. Our study is the first to investigate the importance of DeltaNLR in predicting ARF development after CABG operations. DeltaNLR 1 and 2 values calculated in our study were determined as independent predictors to predict postoperative ARF (OR: 3.090, CI 95\%: 1.698-6.430, $P<.001$, OR: 1.676 , CI 95\%: 1.322-2.764, $P=.003$, respectively).

Other known risk factors for ARF in the postoperative period are older age, the use of CPB systems, preoperative increased creatinine values, and increased preoperative blood product use. Atherosclerotic foci increase with advanced age, which may cause renal ischemic events, due to atheroembolism along with the use of CPB systems. Similar to the literature, we found a significant relationship between advanced age and ARF in our study [Ried 2011; Amini 2019]. Increased blood product use results in increased hemolysis, which can lead to ARF by increasing the free iron content in the blood [Freeland 2015]. In our study, we found a significant relationship between increased transfusion and ARF, consistent with the literature [Karkouti 2011]. However, there are studies in the literature stating that blood transfusion may pose risks only in anemic patients [Granata 2012]. Drugs used in inotropic support may cause renal damage, due to vasoconstrictor effects. Although it was not identified as an independent predictor in the study by Parlar et al, a significant correlation was found between ARF development and inotrope use [Parlar 2018]. In our study, we determined the use of inotropic support as an independent predictor, although its clinical significance is minor (OR: 0.678).

In $\mathrm{CABG}$ operations performed using cardiopulmonary bypass systems, hemolysis occurs due to contact of blood with foreign surfaces. In addition, microatheroembolic states can develop due to cross-clamp use [Granata 2012]. For this reason, the risk of ARF increases. Hemoglobin, which freely circulates because of hemolysis, consumes haptoglobin in the bloodstream and catalyzes free radical production, forming a precipitate with Tamms-Horsfall proteins in the kidney collection system. Simultaneously, vasoconstriction occurs in kidney arterioles due to increased nitric oxide consumption, leading to a damage in the kidneys [Ronco 2007]. However, there is no complete consensus on the effect of the CPB system on ARF in the literature. In a meta-analysis performed in 2014, it was shown that ARF was detected $40 \%$ less in patients who received CABG without pump [Schopka 2014]. Although we did not find the total perfusion time as an independent predictor in our study, we found a correlation with ARF in significant univariate analysis (OR: 0.856, CI 95\%: 0.578-0.994, $P=.016)$.

Study limitations: The most important limitations of our study are its single center, retrospective design, and small number of patients. In addition, our study includes isolated
CABG patients. Further multi-centered novel studies with large patient series are needed to investigate the importance of this issue for all operations using cardiopulmonary bypass systems.

\section{CONCLUSIONS}

In this study, we have shown that peroperative NLR changes can be used as an effective parameter to predict ARF developing following CABG operations with CPB. The use of preoperative change in NLR, rather than NLR value itself, which is a highly investigated parameter with meta-analyses whose predictive value in cardiovascular diseases is accepted widely, may be a more valuable parameter for predicting postoperative ARF.

\section{REFERENCES}

Amini S, Najafi MN, Karrari SP, et al. 2019. Risk Factors and Outcome of Acute Kidney Injury after Isolated CABG Surgery: a Prospective Cohort Study. Braz J Cardiovasc Surg. 34(1):70-75.

Bonventre JV, Yang L. 2011. Cellular pathophysiology of ischemic acute kidney injury. J Clin Invest. 121:4210-4221.

Engin M, Aydin C. 2020. Investigation of the Effect of HATCH Score and Coronary Artery Disease Complexity on Atrial Fibrillation After OnPump Coronary Artery Bypass Graft Surgery. Med Princ Pract. May 18.

Erdolu B, As AK, Engin M. 2020. The Relationship between the HATCH Score, Neutrophil to Lymphocyte Ratio and Postoperative Atrial Fibrillation Afer O -Pump Coronary Artery Bypass Graf Surgery. Heart Surg Forum. 23(1):E88-E92.

Freeland K, Hamidian Jahromi A, Duvall LM, et al. 2015. Postoperative blood transfusion is an independent predictor of acute kidney injury in cardiac surgery patients. J Nephropathol. 4(4):121-6.

Granata A, Insalaco M, Di Pietro F, et al. 2012. Atheroembolism renal disease: diagnosis and etiologic factors. Clin Ter. 163(4):313-22.

Karkouti K. 2012. Transfusion and risk of acute kidney injury in cardiac surgery. Br J Anaesth. 109(suppl 1):i29-i38.

Karkouti K, Wijeysundera DN, Yau TM, et al. 2011. Influence of erythrocyte transfusion on the risk of acute kidney injury after cardiac surgery di ers in anemic and nonanemic patients. Anesthesiology. 115(3):523-30.

Kim WH, Park JY, Ok SH, et al. 2015. Association Between the Neutrophil/Lymphocyte Ratio and Acute Kidney Injury After Cardiovascular Surgery: A Retrospective Observational Study. Medicine (Baltimore). 94(43):e1867.

Laffey JG, Boylan JF, Cheng DC. 2002. The systemic inflammatory response to cardiac surgery: Implications for the anesthesiologist. Anesthesiology. 97: 215-252.

Lee SI, Lee SY, Choi CH, et al. 2018. Relation between changes in red blood cell distribution width after coronary artery bypass grafting and early postoperative morbidity. J Thorac Dis.10(7):4244-4254.

Li C, Zhang F, Shen Y, et al. 2017. Impact of Neutrophil to Lymphocyte Ratio (NLR) Index and Its Periprocedural Change (NLRD) for Percutaneous Coronary Intervention in Patients With Chronic Total Occlusion. Angiology. 68(7):640-646. 
Parlar H, SaSkın H. 2018. Are Pre and Postoperative Platelet to Lymphocyte Ratio and Neutrophil to Lymphocyte Ratio Associated with Early Postoperative AKI Following CABG? Braz J Cardiovasc Surg. 33(3):233-241.

Ried M, Puehler T, Haneya A, et al. 2011. Acute kidney injury in septuaand octogenarians after cardiac surgery. BMC Cardiovasc Disord. 11(1):52.

Ronco CBR, Kellum JA. 2007. Acute kidney injury. Contrib Nephrol. $156: 340-53$

Schopka S, Diez C, Camboni D, et al. 2014. Impact of cardiopulmonary bypass on acute kidney injury following coronary artery bypass grafting: a matched pair analysis. J Cardiothorac Surg. 9(1):20.

Seong YW, Han SJ, Jung W, et al. 2019. Perioperative change in neutrophil-to-lymphocyte ratio (NLR) is a prognostic factor in patients with completely resected primary pulmonary sarcomatoid carcinoma. J Thorac Dis. 11(3):819-826.
Sharfuddin AA, Molitoris BA. 2011. Pathophysiology of ischemic acute kidney injury. Nat Rev Nephrol. 7:189-200.

Takaki S, Shehabi Y, Pickering JW, et al. 2015. Perioperative change in creatinine following cardiac surgery with cardiopulmonary bypass is useful in predicting acute kidney injury: a single-centre retrospective cohort study. Interact Cardiovasc Thorac Surg. 21(4):465-9.

Tumer NB, Kunt AT, Gunaydin S. 2019. Preoperative Urinary pH is Associated with Acute Kidney Injury Afer Cardiac Surgery in Non-Diabetic Patients. Heart Surg Forum. 22(5):E456-E461.

Weedle RC, Da Costa M, Veerasingam D, Soo AWS. 2019. The use of neutrophil lymphocyte ratio to predict complications post cardiac surgery. Ann Transl Med. 7(23):778.

Yilmaz H, Cakmak M, Inan O, et al. 2015. Can neutrophil-lymphocyte ratio be independent risk factor for predicting acute kidney injury in patients with severe sepsis? Ren Fail. 37:225-229. 